\title{
Placas eritematoedematosas en el tronco
}

\section{Erythematous-edematous plaques on the trunk}

\section{Florencia Guillermina Martínez ${ }^{1}$, Paula Johana Barba², Karina Latorre ${ }^{3}$ y Mariana Hurtado ${ }^{4}$}

1 Médica Concurrente

2 Médica Residente

${ }^{3}$ Médica Dermatóloga de Planta

${ }^{4}$ Jefa del Servicio

Servicio de Dermatología, Hospital Interzonal General de Agudos Prof. Dr. Rodolfo Rossi, La Plata, Provincia de Buenos Aires, Argentina

\section{CASO CLÍNICO}

Una mujer de 53 años, con antecedentes personales de diabetes mellitus y cáncer de mama metastásico en tratamiento con docetaxel y gemcitabina, consultó por la aparición de una dermatosis en el tronco, de 5 días de evolución, asintomática, asociada a registros febriles. Refería que hacía un año, luego de la radioterapia, había presentado un cuadro similar localizado solo sobre el sitio irradiado, tratado con corticosteroides orales, con buena respuesta.
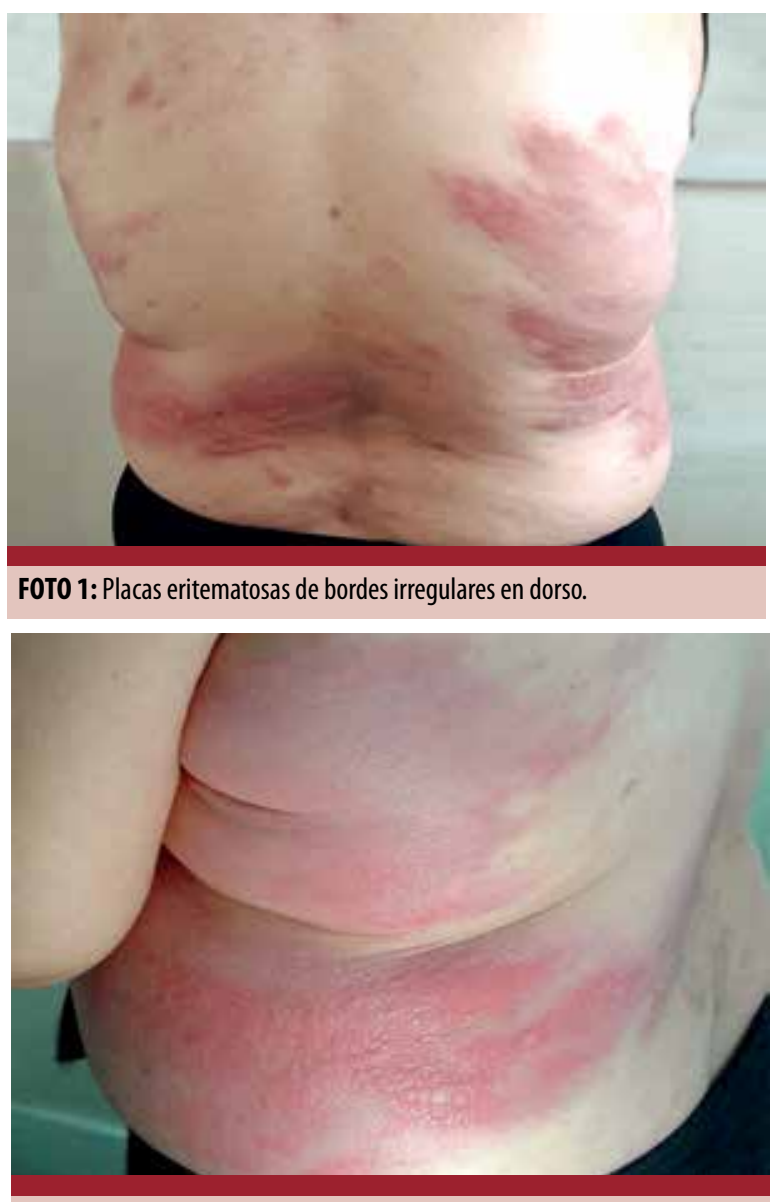

F0T02:. Placa eritematoedematosa en la región lumbar izquierda.
Contacto del autor: Florencia Guillermina Martínez

E-mail: florenciaguillerminamartinez@gmail.com

Fecha de trabajo recibido: $27 / 5 / 2020$

Fecha de trabajo aceptado: 22/2/2021

Conflicto de interés: las autoras declaran que no existe conflicto de interés.

Dermatol. Argent. 2020, 27 (1): 37-38

En el examen físico, presentaba placas eritematoedematosas extensas, de disposición asimétrica y bordes irregulares, que ocupaban la región anterior y posterior del tronco (Fotos 1 y 2 ).

Se realizó una biopsia cutánea para el estudio histopatológico, que informó: marcado edema en la dermis superficial junto con un intenso infiltrado inflamatorio intersticial y angiocéntrico, con predominio neutrofílico, sin presencia de vasculitis (Fotos 3 y 4 ).

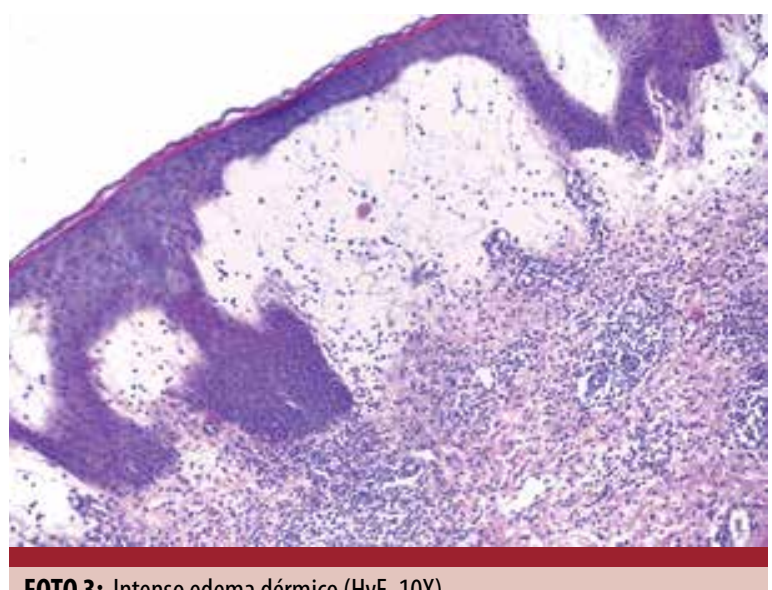

F0T0 3: Intenso edema dérmico (HyE, 10X).

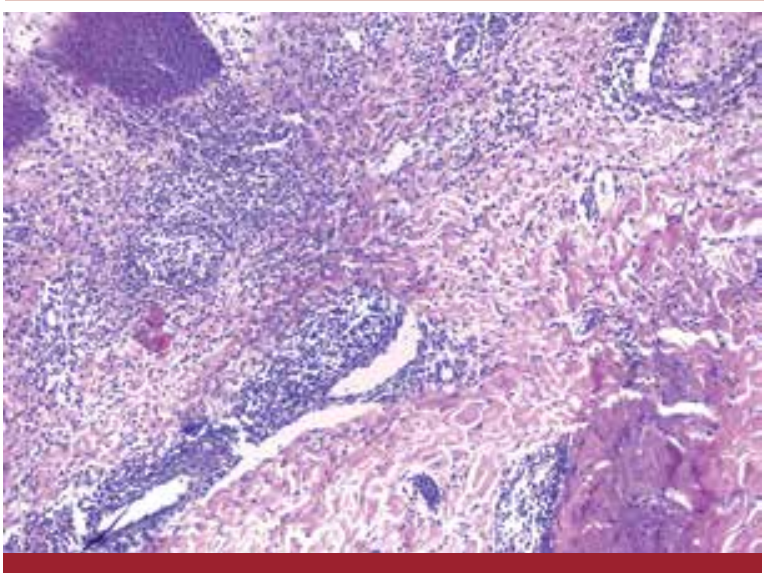

F0T0 4: Infiltrado inflamatorio neutrofilico intersticial y angiocéntrico (HyE, 10X). 
Se solicitó una analítica sanguínea, que arrojó positividad en los siguientes parámetros: leucocitos $11.200 / \mathrm{ml}$ (neutrófilos 91,4\%, linfocitos 4,9\%, monocitos 3,7\%) y velocidad de sedimentación globular (VSG) $50 \mathrm{~mm} /$ hora.

\section{DIAGNÓSTICO}

Síndrome de Sweet paraneoplásico.

\section{COMENTARIOS}

El síndrome de Sweet o dermatosis neutrofílica febril aguda es una enfermedad inflamatoria cutánea poco frecuente, caracterizada por fiebre, leucocitosis con neutrofilia, pápulas o placas eritematosas dolorosas y un denso infiltrado neutrofílico en la dermis papilar en ausencia de infección ${ }^{1}$.

Se define por la presencia de dos criterios mayores: inicio abrupto de placas o nódulos eritematosos dolorosos e infiltrado dérmico predominantemente neutrofílico sin vasculitis leucocitoclásica; y cuatro criterios menores: asociación con infección previa, vacunación, enfermedades inflamatorias, neoplasias, consumo de fármacos o drogas, embarazo, presencia de fiebre $>38{ }^{\circ} \mathrm{C}$, signos y síntomas constitucionales, valores de laboratorio alterados (VSG $>20 \mathrm{~mm} / \mathrm{h}$, proteína C-reactiva positiva, leucocitosis $>8.000 / \mathrm{ml}$, $>70 \%$ de neutrófilos) y excelente respuesta al tratamiento con corticosteroides sistémicosi, ${ }^{1,2}$.

El síndrome de Sweet puede clasificarse en clásico, asociado a malignidad o inducido por fármacos ${ }^{3}$.

El síndrome asociado a malignidad o paraneoplásico representa un 3-35\% de los casos $^{3}$. Las neoplasias

\section{BIBLIOGRAFÍA}

1. Marzano AV, Borghi A, Wallach D, Cugno M. A Comprehensive Review of Neutrophilic Diseases. Clin Rev Allerg Immunol 2018;54:114-130.

2. Marcoval J, Martín-Callizo C, Valentí-Medina F, Bonfill-Ortí M, et ál. Sweet syndrome: long-term follow-up of 138 patients. Clin Exp Dermatol 2016;41:741-746.

3. Nelson CA, Stephen S, Ashchyan HJ, James WD, et ál. Neutrophilic dermatoses: Pathogenesis, Sweet syndrome, neutrophilic eccrine hidradenitis, and Behçet disease. J Am Acad Dermatol 2018;79:987-1006.

4. Coskun U, Gunel N, Senol E, Ilter N, et ál. A case of Sweet's mieloproliferativas o trastornos mielodisplásicos, en especial la leucemia mieloide aguda y el síndrome mielodisplásico, se han implicado con mayor frecuencia que las neoplasias malignas sólidas, en una proporción de 4:1 a 8:13. Los tumores sólidos representan el 23\% de los casos, entre ellos, los tumores genitourinarios como los de testículo y de próstata, los de ovario y mama y los adenocarcinomas de colon y rectales son los que se han publicado con mayor frecuencia ${ }^{1}$.

Ciertas características clínico-patológicas se han relacionado con malignidad: edad avanzada, morfología vesicoampollar, lesiones más extensas o generalizadas, compromiso mucoso, valores elevados de VSG y, a menudo, anemia o trombocitopenia asociada ${ }^{1,3}$.

Con especial referencia al síndrome de Sweet paraneoplásico asociado al cáncer de mama, se han publicado varios casos hasta la fecha. Las lesiones pueden aparecer antes o al mismo tiempo que las metástasis y, también, como una dermatosis incidental que ocurre en el brazo linfedematoso luego de una mastectomía homolateral y disección de ganglios linfáticos ${ }^{4,5}$. Por último, se describió que la radioterapia como tratamiento del cáncer de mama puede inducir la enfermedad localizada, con la erupción de lesiones en el campo de irradiación después de meses o años de finalizada esta ${ }^{6}$.

Como conclusión, el síndrome de Sweet paraneoplásico debe tenerse en cuenta en los pacientes de edad avanzada con lesiones extensas y morfología vesicoampollar. Dada su frecuente asociación con una enfermedad recurrente o metastásica, se debe tener alta sospecha clínica y realizar los estudios correspondientes, ya que augura una evolución tórpida y un mal pronóstico.

syndrome developed after the treatment of herpes simplex infection in a metastatic breast cancer patient. J Cutan Pathol 2002;29:301-304.

5. Cohen PR. Proton pump inhibitor-induced Sweet's syndrome: report of acute febrile neutrophilic dermatosis in a woman with recurrent breast cancer. Dermatol Pract Concept 2015;5:113-119.

6. Tang HKC, Lee DYH, Thompson I, Ingram JR, et ál. Delayed and localized Sweets syndrome after breast cancer radiotherapy. Breast J 2018;24:212-213. 